\title{
The European Society of Gynaecological Oncology/European Society for Radiotherapy and Oncology/European Society of Pathology Guidelines for the Management of Patients with Cervical Cancer
}

\author{
David Cibula $^{1}$ - Richard Pötter ${ }^{2}$. François Planchamp ${ }^{3}$ - Elisabeth Avall-Lundqvist ${ }^{4}$ - Daniela Fischerova ${ }^{1}$. \\ Christine Haie-Meder ${ }^{5}$. Christhardt Köhler ${ }^{6}$. Fabio Landoni ${ }^{7}$. Sigurd Lax ${ }^{8}$. Jacob Christian Lindegaard ${ }^{9}$. \\ Umesh Mahantshetty ${ }^{10}$ - Patrice Mathevet ${ }^{11}$ - W. Glenn McCluggage ${ }^{12}$ - Mary McCormack ${ }^{13}$ - Raj Naik ${ }^{14}$. \\ Remi Nout ${ }^{15}$. Sandro Pignata ${ }^{16}$. Jordi Ponce ${ }^{17}$. Denis Querleu ${ }^{3}$. Francesco Raspagliesi $^{18}$. \\ Alexandros Rodolakis ${ }^{19} \cdot$ Karl Tamussino $^{20}$ • Pauline Wimberger ${ }^{21}$ • Maria Rosaria Raspollini ${ }^{22}$
}

Received: 12 January 2018 / Accepted: 12 January 2018 / Published online: 4 May 2018

(C) European Society for Gynaecological Oncology, European Society for Radiotherapy and Oncology, and European Society of Pathology. Published by Springer-Verlag GmbH Berlin Heidelberg 2018

\begin{abstract}
Background: Despite significant advances in the screening, detection, and treatment of preinvasive cervical lesions, invasive cervical cancer is the fifth most common cancer in European women. There are large disparities in Europe and worldwide in the incidence, management, and mortality of cervical cancer.

Objective: The European Society of Gynecological Oncology (ESGO), the European Society for Radiotherapy and Oncology (ESTRO), and the European Society of Pathology (ESP) jointly develop clinically relevant and evidence-based guidelines in order to improve the quality of care for women with cervical cancer across Europe and worldwide.

Methods: The ESGO/ESTRO/ESP nominated an international multidisciplinary development group consisting of practicing clinicians and researchers who have demonstrated leadership and expertise in the care and research of cervical cancer (23 experts across Europe). To ensure that the guidelines are evidence based, the current literature identified from a systematic search was reviewed and critically appraised. In the absence of any clear scientific evidence, judgment was based on the professional experience and consensus of the development group. The guidelines are thus based on the best available evidence and expert
\end{abstract}

These guidelines statements were developed by ESGO, ESTRO and ESP and are published in the International Journal of Gynecological Cancer, Radiotherapy \& Oncology and Virchows Archiv.

Initiated through the European Society of Gynaecological Oncology (ESGO), the decision to develop multidisciplinary guidelines has been made jointly by ESGO, the European Society for Radiotherapy and Oncology (ESTRO), and the European Society of Pathology (ESP). ESGO has provided administrative support. The only external funding source was a grant from the Institut National du Cancer (France). ESGO, ESTRO, and ESP are nonprofit knowledgeable societies. The Institut National du Cancer is a French government agency.

The development group (including all authors) is collectively responsible for the decision to submit for publication. D.C. (chair), R.P. (cochair), M.R.R. (cochair), and F.P. (methodologist) have written the first draft of the manuscript. All other contributors have actively participated to the development group, given personal input, reviewed the manuscript, and given final approval before submission.

Electronic supplementary material The online version of this article (https://doi.org/10.1007/s00428-018-2362-9) contains supplementary material, which is available to authorized users.

David Cibula

dc@davidcibula.cz 
agreement. Prior to publication, the guidelines were reviewed by 159 international reviewers, selected through ESGO/ESTRO/ ESP and including patient representatives.

Results: The guidelines cover comprehensively staging, management, and follow-up for patients with cervical cancer. Management includes fertility sparing treatment; stage T1a, T1b1/T2a1, clinically occult cervical cancer diagnosed after simple hysterectomy; early and locally advanced cervical cancer; primary distant metastatic disease; cervical cancer in pregnancy; and recurrent disease. Principles of radiotherapy and pathological evaluation are defined.

Keywords Cervical cancer $\cdot$ Guidelines $\cdot$ Management $\cdot$ Staging $\cdot$ Follow-up

The objectives of the guidelines are to improve and to homogenize the management of patients with cervical cancer within a multidisciplinary setting. These guidelines are intended for use by gynecologic oncologists, general gynecologists, surgeons, radiation oncologists, pathologists, medical and clinical oncologists, radiologists, general practitioners, palliative care teams, and allied health professionals. The guidelines aim at covering comprehensively staging, management, and follow-up for patients with cervical cancer. Management includes fertilitysparing treatment (FST); stage T1a, T1b1/T2a1, clinically occult cervical cancer diagnosed after simple hysterectomy; early and locally advanced cervical cancer; primary distant metastatic disease; cervical cancer in pregnancy (CCIP); and recurrent disease. The characteristics of the pathology report that represents a key component in the management of cervical cancer patients are outlined. Principles of current cervical cancer radiotherapy are defined. These guidelines exclude the management of neuroendocrine carcinomas, sarcomas, and other rare histologic subtypes. They also do not include any economic analysis of the strategies.

\section{Responsibilities}

These guidelines are a statement of evidence and consensus of the authors regarding their views of currently accepted approaches to treatment. Any clinician applying or consulting these guidelines is expected to use independent medical judgment in the context of individual clinical circumstances to determine any patient's care or treatment. These guidelines make no representations or warranties of any kind whatsoever regarding their content, use, or application and disclaim any responsibility for their application or use in any way.

\section{Methods}

The guidelines were developed using a 5-step process as defined by the European Society of Gynecological Oncology (ESGO) Guideline Committee (Fig. 1). The strengths of the process include creation of a multidisciplinary international development group, use of scientific evidence and/or international expert consensus to support the guidelines, and use of an international external review process (physicians and patients). This development process involved 3 meetings of the international development group, chaired by Prof David Cibula (Charles University, Prague, Czech Republic), Prof Richard Pötter (Medical University of Vienna, Vienna, Austria), and Prof Maria Rosaria Raspollini (University Hospital, Careggi, Florence, Italy).

\section{Step 1: Nomination of multidisciplinary international development group}

The European Society of Gynecological Oncology/ European Society for Radiotherapy and Oncology (ESTRO)/ European Society of Pathology (ESP) nominated practicing clinicians who are involved in the management of cervical cancer patients and have demonstrated leadership in clinical management of patients through research, administrative responsibilities, and/or committee membership to serve on the expert panel. The objective was to assemble a multidisciplinary panel. It was therefore essential to include professionals from relevant disciplines (surgery, medical oncology, pathology, radiology, gynecology, radiation oncology) to contribute to the validity and acceptability of the guidelines. The experts of the multidisciplinary international development group were required to complete a declaration of interest form and to promptly inform the ESGO council if any change in the disclosed information occurred during the course of the work.

\section{Step 2: Identification of scientific evidence}

To ensure that the statements were evidence based, the current literature was reviewed and critically appraised. A systematic literature review of relevant studies published between January 1997 and January 2017 was carried out using the MEDLINE database (Supplementary Material 1). The literature search was limited to publications in English. Priority was given to highquality systematic reviews, meta-analyses, and randomized controlled trials, but studies of lower levels of evidence were also evaluated. The search strategy excluded editorials, letters, and in vitro studies. The reference list of each identified article 
Fig. 1 Development process

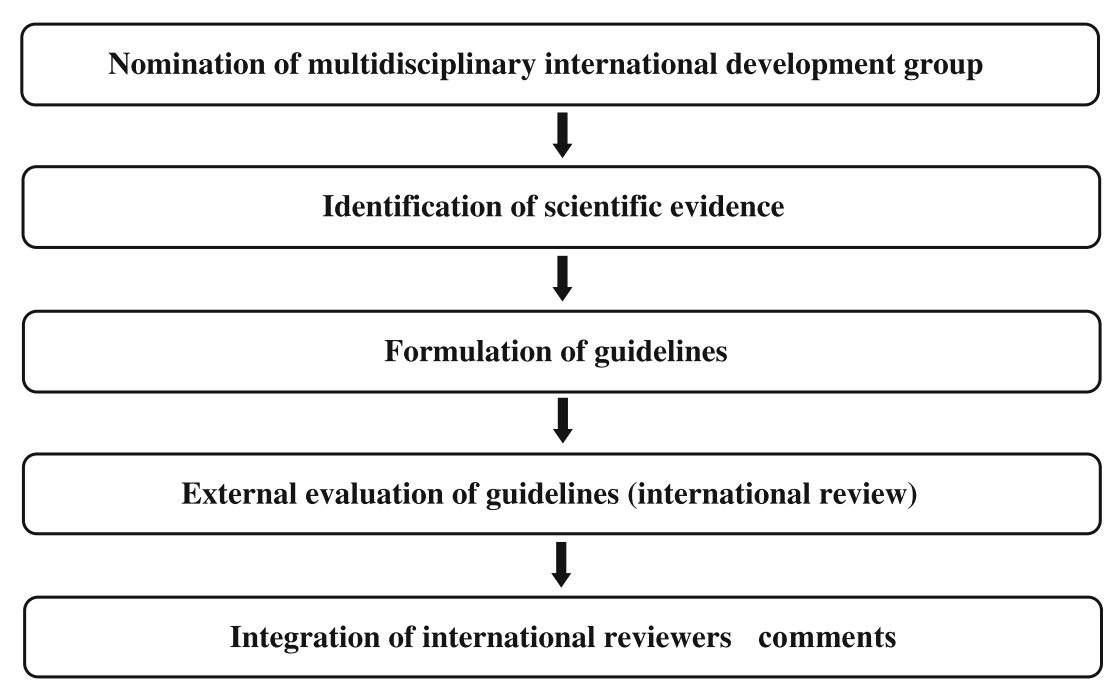

was reviewed for other potentially relevant articles. The bibliography was also supplemented by additional references provided by the international development group. Another bibliographic search was carried out to identify previous initiatives using a systematic literature search in MEDLINE database (no restriction in the search period) and a bibliographic search using selected evidence-based medicine Web sites (Supplementary Material 2). After the selection and critical appraisal of the articles whose full list of references is available on the ESGO website, a summary of the scientific evidence was developed.

\section{Step 3: Formulation of guidelines}

The multidisciplinary expert group developed guidelines for staging, FST, management (stage T1a, T1b1/T2a1, clinically occult cervical cancer diagnosed after simple hysterectomy, locally advanced cervical cancer, primary distant metastatic disease, CCIP, and recurrent disease), and follow-up for patients with cervical cancer. If an approach was judged to be acceptable but was not unanimously recognized as a criterionstandard clinical approach, indication was given that it was still subject to discussion and/or evaluation. In the absence of any clear scientific evidence, judgment was based on the professional experience and consensus of the development group. The reliability and quality of the evidence given throughout this article have been graded following the Scottish intercollegiate guidelines network grading system (Table 1). Grading of principles of radiotherapy and principles of pathological evaluation according to this grading system is challenging and is beyond the scope of these guidelines.

\section{Step 4: External evaluation of the guidelines - International review}

The ESGO/ESTRO/ESP consulted a large panel of practicing clinicians who are involved in the management of cervical cancer patients. Cervical cancer patients were also included. The objective was to assemble a multidisciplinary panel. The 159 international reviewers were independent from the multidisciplinary expert group. International reviewers were asked to evaluate each guideline according to their relevance and feasibility in clinical practice (only physicians). Quantitative and qualitative evaluations of the guidelines were performed. Patients were asked to qualitatively evaluate each guideline (according to their experience, preferences, feelings, etc).

\section{Step 5: Integration of international reviewers comments}

Responses of the 159 external reviewers were pooled and discussed by the international development group to finalize the guidelines.

The complete report for the management of patients with cervical cancer contains the comprehensive literature review (indicating the level of evidence), justifications for selected recommendations, and the guidelines (indicating the grading of the guidelines) and algorithms. This complete report is available online at ESGO/ESTRO/ESP Web sites (https:// guidelines.esgo.org/, https://www.estro.org/, https://www. esp-pathology.org/publications/guidelines.html, https://www. esp-pathology.org/working-groups/esp-working-groups/ gynecologic-pathology.html).

\section{General recommendations}

- Treatment planning should be made on a multidisciplinary basis (generally at a tumor board meeting) and based on the comprehensive and precise knowledge of prognostic and predictive factors for oncological outcome, morbidity, and quality of life. 
Table 1 Grades of recommendations

A At least 1 meta-analysis, systematic review, or RCT rated as 1++, and directly applicable to the target population; or a body of evidence consisting principally of studies rated as $1+$, directly applicable to the target population, and demonstrating overall consistency of results

B A body of evidence including studies rated as $2++$, directly applicable to the target population, and demonstrating overall consistency of results; or extrapolated evidence from studies rated as $1++$ or $1+$

C A body of evidence including studies rated as 2+, directly applicable to the target population and demonstrating overall consistency of results; or extrapolated evidence from studies rates as $2++$

D Evidence level 3 or 4 or extrapolated evidence from studies rated as $2+$

$\checkmark \quad$ Recommended best practice based on the clinical experience of the guideline development group

$1++=$ High-quality meta-analyses, systematic reviews of randomized controlled trials (RCTs), or RCTs with a very low risk of bias; $1+=$ well-conducted meta-analyses, systematic reviews, or RCTs with a low risk of bias; $2++=$ high-quality systematic reviews of case-control or cohort studies or highquality case-control or cohort studies with a very low risk of confounding or bias and a high probability that the relationship is causal; $2+=$ wellconducted casecontrol or cohort studies with a low risk of confounding or bias and a moderate probability that the relationship is causal; $3=$ nonanalytic studies, for example, case reports, case series; 4 = expert opinion.

- Patients should be carefully counseled on the suggested treatment plan and potential alternatives, including risks and benefits of all options.

- Treatment should be undertaken by a dedicated team of specialists in the diagnosis and management of gynecologic cancers.

\section{Staging}

\section{TNM classification and International Federation of Gynecology and Obstetrics}

- Patients with cervical cancer should be staged according to the TNM classification. Clinical staging (International Federation of Gynecology and Obstetrics [FIGO]) should also be documented (Table 2).

- TNM should be based on a correlation of various modalities (integrating physical examination, imaging and pathology) after discussion in a multidisciplinary forum (grade $\mathrm{C}$ ).

- The method used to determine tumor status (T), lymph node status $(\mathrm{N})$, and systemic status $(\mathrm{M})$, that is, clinical (c), imaging (i), and/or pathological (p) should be recorded/

- Lymph node metastases should be classified according to the TNM classification (see PRINCIPLES OF PATHOLOGICAL EVALTUATION).

\section{Prognostic factors}

Proper documentation of the following major tumor-related prognostic factors is recommended (grade A):

- $\quad$ TNM and FIGO stage, including a maximum tumor size and detailed description of extracervical tumor extension and nodal involvement (number, size, location)

- Pathological tumor type
- Depth of cervical stromal invasion and a minimum thickness of uninvolved cervical stroma

- Presence or absence of lymphovascular space involvement (LVSI)

- Presence or absence of distant metastases

\section{Local clinical and radiological diagnostic workup}

- Pelvic examination and biopsy +/- colposcopy are mandatory components to diagnose cervical cancer.

- Mandatory initial workup for assessment of pelvic tumor extent and to guide treatment options is pelvic magnetic resonance imaging (MRI) (grade B). Endovaginal/ transrectal ultrasound is an option if performed by a properly trained sonographer.

- Cystoscopy or rectoscopy may be considered to provide a biopsy if suspicious lesions in the urinary bladder or rectum are documented on MRI or ultrasound.

\section{Nodal/distant diagnostic workup}

- In early stage (T1a, T1b1, T2a1), surgical/pathological staging of pelvic lymph nodes is the criterion standard to assess the prognosis and guide treatment (except of T1a1 and no LVSI) (grade B).

- In locally advanced cervical cancer (T1b2 and higher [except T2a1]) or in early-stage disease with suspicious lymph nodes on imaging, positron emission tomography-computed tomography (PET-CT), or chest/ abdomen CTis recommended for assessment of nodal and distant disease (grade B).

- Positron emission tomography-CT is the preferred option for treatment planning before chemoradiotherapy with curative intent (grade B).

- Para-aortic lymph node dissection, at least up to inferior mesenteric artery, may be considered in locally advanced 
Table 2 FIGO staging and TNM classification

\begin{tabular}{|c|c|c|}
\hline T Category* & FIGO Stage $\dagger$ & Definition \\
\hline TX & & Primary tumor cannot be assessed \\
\hline T0 & & No evidence of primary tumor \\
\hline $\mathrm{T} 1$ & I & Cervical carcinoma confined to the uterus (extension to corpus should be disregarded) \\
\hline T1a & IA & $\begin{array}{l}\text { Invasive carcinoma diagnosed only by microscopy. Stromal invasion with a maximum depth of } 5.0 \mathrm{~mm} \text { measured } \\
\text { from the base of the epithelium and a horizontal spread of } \leq 7.0 \mathrm{~mm} \text {; vascular space involvement, venous or } \\
\text { lymphatic, does not affect classification }\end{array}$ \\
\hline T1a1 & IA1 & Measured stromal invasion of $\leq 3.0 \mathrm{~mm}$ in depth and $\leq 7.0 \mathrm{~mm}$ in horizontal spread \\
\hline T1a2 & IA2 & Measured stromal invasion of $>3.0 \mathrm{~mm}$ and not $>5.0 \mathrm{~mm}$, with a horizontal spread of $\leq 7.0 \mathrm{~mm}$ \\
\hline $\mathrm{T} 1 \mathrm{~b}$ & IB & $\begin{array}{l}\text { Clinically visible lesion confined to the cervix or microscopic lesion greater than T1a2/IA2. } \\
\text { Includes all macroscopically visible lesions, even those with superficial invasion }\end{array}$ \\
\hline T1b1 & IB1 & Clinically visible lesion $\leq 4.0 \mathrm{~cm}$ in greatest dimension \\
\hline T1b2 & IB2 & Clinically visible lesion $>4.0 \mathrm{~cm}$ in greatest dimension \\
\hline $\mathrm{T} 2$ & II & Cervical carcinoma invading beyond the uterus but not to the pelvic wall or to lower third of the vagina \\
\hline $\mathrm{T} 2 \mathrm{a}$ & IIA & Tumor without parametrial invasion \\
\hline T2a1 & IIA1 & Clinically visible lesion $\leq 4.0 \mathrm{~cm}$ in greatest dimension \\
\hline $\mathrm{T} 2 \mathrm{a} 2$ & IIA2 & Clinically visible lesion $>4.0 \mathrm{~cm}$ in greatest dimension \\
\hline $\mathrm{T} 2 \mathrm{~b}$ & IIB & Tumor with parametrial invasion \\
\hline $\mathrm{T} 3$ & III & $\begin{array}{l}\text { Tumor extending to the pelvic sidewallt and/or involving the lower third of the vagina and/or causing } \\
\text { hydronephrosis or nonfunctioning kidney }\end{array}$ \\
\hline T3a & IIIA & Tumor involving the lower third of the vagina but not extending to the pelvic wall \\
\hline $\mathrm{T} 3 \mathrm{~b}$ & IIIB & Tumor extending to the pelvic wall and/or causing hydronephrosis or nonfunctioning kidney \\
\hline \multirow[t]{2}{*}{ T4 } & IVA & $\begin{array}{l}\text { Tumor invading the mucosa of the bladder or rectum and/or extending beyond the true pelvis (bullous edema is } \\
\text { not sufficient to classify a tumor as T4) }\end{array}$ \\
\hline & IVB & Tumor invading distant organs \\
\hline
\end{tabular}

*Union for International Cancer Control (UICC). Eighth edition of the UICC TNM Classification of Malignant Tumors (2016)

$\dagger$ Pecorelli, S. Revised FIGO staging for carcinoma of the vulva, cervix, and endometrium. Int J Gynaecol Obstet 105, 103-104 (2009)

$\$$ The pelvic sidewall is defined as the muscle, fascia, neurovascular structures, and skeletal portions of the bony pelvis

Pecorelli S, Zigliani L, Odicino F. Revised FIGO staging for carcinoma of the cervix. Int J Gynaecol Obstet 2009;105:107-108. Pecorelli S

Corrigendum to "Revised FIGO staging for carcinoma of the vulva, cervix, and endometrium." Int J Gynaecol Obstet 2010;108:176

cervical cancer with negative para-aortic lymph nodes on imaging for staging purposes (grade $\mathrm{C}$ ).

- Equivocal extrauterine disease is to be considered for biopsy to confirm or rule out metastatic disease and to avoid inappropriate treatment. Tru-Cut (core-cut) biopsy is the preferred option than fine-needle aspiration biopsy because it allows histological assessment of the tissue.

\section{Management of stage T1a}

\section{Diagnosis of stage $\mathrm{T} 1 \mathrm{a}$ disease}

- Diagnosis of T1a cancer should be based on a conization (or excision) specimen examined by an expert pathologist. Management must be based on an expert pathology review, with accurate measurement of the maximum horizontal 2 dimensions, depth of invasion, margin status, coexisting pathology, and reliable assessment of LVSI.
- Loop or laser conization is preferable to cold-knife conization in women desiring fertility preservation. Maximum care should be taken to provide an intact (unfragmented) specimen with minimal thermal artifact. The cone specimen should be oriented for the pathologist.

- Surgical margins of the cone specimen should be clear of both invasive and preinvasive disease (except for preinvasive disease in ectocervix) (grade C).

\section{Management of stage T1a1 disease}

- Management of patients with stage T1al disease should be individualized depending on the age, the desire for fertility preservation, and the presence or absence of LVSI.

- In case of positive margins (except for preinvasive disease in ectocervix), a repeat conization should be performed to rule out more extensive invasive disease.

- Lymph node staging is not indicated in T1a1 LVSInegative patients but can be considered in T1a1 LVSI- 
positive patients. Sentinel lymph node biopsy (without additional pelvic lymph node dissection) is an acceptable method of lymph node staging (grade B).

- Conization can be considered a definitive treatment as hysterectomy does not improve the outcome (grade C).

- Radical surgical approaches such as radical hysterectomy or parametrectomy represent overtreatment for patients with T1a1 disease (grade C).

\section{Management of stage $\mathrm{T} 1 \mathrm{a} 2$ disease}

- In patients with stage $\mathrm{T} 1 \mathrm{a} 2$ disease, conization alone or simple hysterectomy is an adequate treatment (grade C).

- Parametrial resection is not indicated (grade C).

- Lymph node staging can be considered in LVSI-negative patients but should be performed in LVSI-positive patients. Sentinel lymph node biopsy alone (without additional pelvic lymph node dissection) appears to be an acceptable method of LN staging (grade B).

- Routine completion of hysterectomy is not recommended after conservative management of stage T1a disease.

\section{Management of stages $\mathrm{T} 1 \mathrm{~b} 1 / \mathrm{T} 2 \mathrm{a} 1$}

\section{General recommendation}

- Treatment strategy should aim for the avoidance of combining radical surgery and radiotherapy because of the highest morbidity after combined treatment (grade B).

\section{Negative lymph nodes on radiological staging - Surgical treatment}

- Radical surgery by a gynecologic oncologist is the preferred treatment modality. Minimally invasive approach is favored (grade B).

- The standard lymph node staging procedure is systematic pelvic lymphadenectomy. Sentinel node biopsy before pelvic lymphadenectomy is strongly recommended. Combination of blue dye with radiocolloid or use of indocyanine green alone is the recommended technique (grade B).

- Lymph node assessment should be performed as the first step of surgical management. Intraoperative assessment of lymph node status (frozen section) is recommended. All sentinel nodes from both sides of the pelvis and/or any suspicious lymph nodes should be sent for frozen section. If sentinel node is not detected, intraoperative assessment of the pelvic lymph nodes should be considered.

- If intraoperative lymph node assessment is negative or not done, systematic pelvic lymph node dissection should be performed. At present, sentinel node biopsy alone cannot be recommended outside prospective clinical trials. Systematic lymph node dissection should include the removal of lymphatic tissue from regions with the most frequent occurrence of positive lymph nodes (sentinel nodes) including obturator fossa, external iliac regions, common iliac regions bilaterally, and presacral region. Distal external iliac lymph nodes (so-called circumflex iliac lymph nodes) should be spared if they are not macroscopically suspicious.

- The type of radical hysterectomy (extent of parametrial resection, type A-C2) should be based on the presence of prognostic risk factors identified preoperatively (Table 3). Major prognostic factors for oncological outcome as tumor size, maximum stromal invasion, and LVSI are used to categorize patients at high, intermediate, and low risk of treatment failure. Complete description of the template used for radical hysterectomy should be present in the surgical report. The 2017 modification of the QuerleuMorrow classification is recommended as a tool (Table 4).

- Ovarian preservation should be offered to premenopausal patients with squamous cell carcinoma and usual-type (human papillomavirus [HPV] related) adenocarcinoma. Bilateral salpingectomy should be considered.

- If lymph node involvement is detected intraoperatively including macrometasteses, further pelvic lymph node dissection and radical hysterectomy should be avoided. Patients should be referred for definitive chemoradiotherapy. Para-aortic lymph node dissection, at least up to inferior mesenteric artery, may be considered for staging purposes (grade C).

- If a combination of risk factors is known at diagnosis, which would require an adjuvant treatment, definitive radiochemotherapy and brachytherapy can be considered without previous radical pelvic surgery. Pelvic lymph node dissection, at least up to inferior mesenteric artery, may be considered in patients with negative para-aortic lymph node on imagine (grade C).

\section{Negative lymph nodes on radiological staging - Alternative treatment options}

- Definitive radiotherapy including brachytherapy represents effective alternative treatment (see PRINCIPLES OF RADIOTHERAPY). It can be considered in particular in case of unfavorable prognostic and predictive factors for oncological and morbidity outcome (grade C).

- For high risk and intermediate risk, preoperative brachytherapy followed by surgery (type A) is used in a limited number of centers. It is an acceptable alternative option only in teams experienced in this approach.

- Neoadjuvant chemotherapy followed by surgery is not recommended (grade $\mathrm{C}$ ). 
Table 3 Risk groups according to prognostic factors: suggested type(s) of radical hysterectomy

\begin{tabular}{lllll}
\hline Risk Group & Tumor Size & LVSI & Stromal Invasion & Type of Radical Hysterectomy* \\
\hline Low risk & $<2 \mathrm{~cm}$ & Negative & Inner 1/3 & B1 (A) \\
Intermediate risk & $\geq 2 \mathrm{~cm}$ & Negative & Any & B2 (C1) \\
& $<2 \mathrm{~cm}$ & Positive & Any & $\mathrm{C} 1(\mathrm{C} 2)$ \\
High risk & $\geq 2 \mathrm{~cm}$ & Positive & Any & \\
\hline
\end{tabular}

*According to the Querleu-Morrow classification (Table 4)

\section{Positive pelvic lymph nodes on radiological staging}

- In patients with unequivocally involved pelvic lymph nodes on imaging, definitive chemoradiotherapy is recommended (see PRINCIPLES OF RADIOTHERAPY). Para-aortic lymph node dissection, at least up to inferior mesenteric artery, may be considered in patients with negative para-aortic lymph nodes on imaging (grade $\mathrm{C}$ ).

- Debulking of suspicious pelvic lymph nodes may be considered.

\section{Adjuvant treatment}

- Adjuvant radiotherapy should be considered in the presence of combination of risk factors at final pathology such as tumor size, LVSI, and depth of stromal invasion (grade B).

- When in these situations an adequate type of radical hysterectomy has been performed (Table 4), observation is an alternative option, especially in teams experienced in this approach.
After primary radical surgery, adjuvant chemoradiotherapy is indicated in the following groups of patients (see PRINCIPLES OF RADIOTHERAPY) (grade B):

- metastatic involvement of pelvic lymph nodes, including the presence of macrometastases $\mathrm{pN} 1$ or micrometastases $\mathrm{pN} 1$ (mi) in either sentinel node or any other pelvic lymph nodes detected by intraoperative or final pathologic assessment $\Rightarrow$ chemoradiotherapy

- $\quad$ positive surgical margins (vagina/parametria) $\Rightarrow$ chemoradiotherapy, brachytherapy boost may be considered

- parametrial involvement $\Rightarrow$ chemoradiotherapy

\section{Cervical stump cancer}

- Management of cervical stump cancer follows the recommendations for patients without previous subtotal hysterectomy. Adaptation of radiotherapy may be necessary, in particular for brachytherapy.

Table 4 Querleu-Morrow classification*

\begin{tabular}{|c|c|c|c|}
\hline Type of Radical Hysterectomy & Paracervix or Lateral Parametrium & Ventral Parametrium & Dorsal Parametrium \\
\hline Type A & $\begin{array}{l}\text { Halfway between the cervix and ureter } \\
\text { (medial to the ureter, ureter identified } \\
\text { but not mobilized) }\end{array}$ & Minimal excision & Minimal excision \\
\hline Type B1 & $\begin{array}{l}\text { At the ureter (at the level of the ureteral } \\
\text { bed, ureter is mobilized from the } \\
\text { cervix and lateral parametrium) }\end{array}$ & $\begin{array}{l}\text { Partial excision of the } \\
\text { vesicouterine ligament }\end{array}$ & $\begin{array}{l}\text { Partial resection of the } \\
\text { rectouterine/rectovaginal } \\
\text { ligament and uterosacral } \\
\text { peritoneal fold }\end{array}$ \\
\hline Type B2 & $\begin{array}{l}\text { Identical to B1 plus paracervical } \\
\text { lymphadenectomy without resection } \\
\text { of vascular/nerve structures }\end{array}$ & $\begin{array}{l}\text { Partial excision of the } \\
\text { vesicouterine ligament }\end{array}$ & $\begin{array}{l}\text { Partial resection of the } \\
\text { rectouterine-rectovaginal } \\
\text { ligament and uterosacral fold }\end{array}$ \\
\hline Type C1 & $\begin{array}{l}\text { At the iliac vessels transversally, } \\
\text { caudal part is preserved }\end{array}$ & $\begin{array}{l}\text { Excision of the vesicouterine } \\
\text { ligament (cranial to the ureter) } \\
\text { at the bladder. Proximal part } \\
\text { of the vesicovaginal ligament } \\
\text { (bladder nerves are dissected } \\
\text { and spared) }\end{array}$ & $\begin{array}{l}\text { At the rectum (hypogastric nerve } \\
\text { is dissected and spared) }\end{array}$ \\
\hline Type C2 & $\begin{array}{l}\text { At the level of the medial aspect of } \\
\text { iliac vessels completely (including } \\
\text { the caudal part) }\end{array}$ & $\begin{array}{l}\text { At the bladder (bladder nerves } \\
\text { are sacrificed) }\end{array}$ & $\begin{array}{l}\text { At the sacrum (hypogastric nerve } \\
\text { is sacrificed) }\end{array}$ \\
\hline Type D & $\begin{array}{l}\text { At the pelvic wall, including resection } \\
\text { of the internal iliac vessels and/or } \\
\text { components of the pelvic sidewall }\end{array}$ & $\begin{array}{l}\text { At the bladder. Not applicable if } \\
\text { part of exenteration }\end{array}$ & $\begin{array}{l}\text { At the sacrum. Not applicable if } \\
\text { part of exenteration }\end{array}$ \\
\hline
\end{tabular}

*Querleu D, Cibula D, Abu-Rustum NR. 2017 Update on the Querleu-Morrow classification of radical hysterectomy. Ann Surg Oncol 2017;24:3406-3412 


\section{Fertility-sparing treatment}

- Before starting FST, consultation at a fertility center is recommended.

- Fertility-sparing treatment should exclusively be undertaken in gynecologic-oncological centers with comprehensive expertise in this kind of oncologic therapy.

- For patients who consider FST, prognostic factors, clinical staging, and preoperative workup do not differ from those who do not consider this (see above).

- Every woman with a desire to spare fertility and histologically proven squamous cell carcinoma or usualtype (HPV-related) adenocarcinoma equal to or less than $2 \mathrm{~cm}$ of the largest diameter should be counseled about the possibility of FST. This consultation should encompass the risk of FST abandonment if there are positive margins or lymph node involvement and oncologic and obstetric risks related to this type of management.

- Fertility-sparing treatment should not be recommended for rare histological subtypes of cervical cancer including neuroendocrine carcinomas and non-HPV-related adenocarcinomas (except for adenoid basal carcinoma), which tend to exhibit aggressive behavior.

- Expert sonography and/or pelvic MRI are recommended imaging tests to measure remaining (after cone biopsy) cervical length and noninvolved cervical length. However, no imaging system can exactly predict the extent of necessary local resection in order to reach sound margins with adequate safety distance.

- Negative pelvic lymph node status is the precondition for any FST. Therefore, pelvic lymph node (sentinel lymph node) staging should always be the first step in each FST procedure. Identification of sentinel lymph node and its ultrastaging is highly recommended because it increases staging accuracy, namely, the identification of micrometastases and small macrometastases. The involvement of suspicious lymph nodes should be confirmed by histology. Intraoperative assessment of lymph node status is highly recommended. All sentinel lymph nodes from both sides of the pelvis or any suspicious lymph nodes should be sent for frozen section. If bilateral sentinel lymph node is not detectable, intraoperative assessment of pelvic lymph nodes should be considered (see MANAGEMENT OF STAGES T1b1/T2a1). Lymph node staging is not indicated in stage T1a1 LVSI negative (grade B).

- In case of intraoperatively proven lymph node involvement, fertility-sparing surgery should be abandoned, and the patient referred to definitive chemoradiotherapy (see above). The specific aim of fertility-sparing surgery must be the resection of invasive tumor with adequate free margins and preservation of the upper part of the cervix.
Intraoperative frozen section is a reliable way of assessing the upper resection margin in trachelectomy specimen and should be considered.

- Conization and simple trachelectomy are adequate fertility-sparing procedures for stages $\mathrm{T} 1 \mathrm{a} 1$ and $\mathrm{T} 1 \mathrm{a} 2$, lymph node-negative, LVSI-negative patients (grade B).

- Radical trachelectomy (type A) can be considered for stages T1a1 and T1a2, lymph node-negative, LVSIpositive patients. Conization or simple trachelectomy is an option (grade B).

- Radical trachelectomy (type B) should be performed for patients with cervical cancer stage T1b1 equal to or less than $2 \mathrm{~cm}$ of the largest diameter, lymph node-negative, LVSI \pm (grade B).

- Intraoperative placement of permanent cerclage should be performed during simple or radical trachelectomy.

- Fertility-sparing treatment in patients with tumors greater than $2 \mathrm{~cm}$ cannot be recommended and is considered as an experimental approach.

- In more advanced cases, different propositions for fertility preservation should be discussed. The goal of the fertility preservation should be to offer the most efficient approach related to the legal aspects of the country while not increasing the oncological risk.

- Any pregnancy following FST should be considered as a high-risk pregnancy, and delivery should be performed in a perinatal center. Following simple or radical trachelectomy with its inherent placement of a permanent cerclage delivery can be performed only by cesarean section.

- Routine hysterectomy after finishing fertility plans is not necessary.

\section{Clinically occult cervical cancer diagnosed after simple hysterectomy}

\section{General recommendations}

- Management of occult disease should be based on expert pathology review and discussed in a multidisciplinary tumor board.

- Prior to making further management decisions, optimal imaging to evaluate the local and regional (nodal) disease status is necessary. Optimal imaging follows the same recommendations as that for nonoccult disease (see above).

- In general, management of occult disease follows the same principles as that of nonoccult disease. Treatment strategy should aim for the avoidance of combining radical surgery and radiotherapy because of the highest morbidity after combined treatment (grade B). 
Management of patients with pT1a1, LVSI \pm , and pT1a1 LVSI negative, with clear margins

- In patients with tumor stage pT1a1 regardless of LVSI status and pT1a2 LVSI negative with clear margins in the hysterectomy specimen, no additional treatment is recommended.

\section{Management of patients with pT1a2 LVSI-positive or $\mathrm{pT} 1 \mathrm{~b} 1$ or $\mathrm{pT2a} 1$, with clear margins}

- In patients with tumor stage pT1a2 LVSI positive or pT1b1 or pT2a1 after simple hysterectomy, potential disease in the parametria and lymph nodes has to be addressed.

- Radiotherapy or chemoradiotherapy is recommended as an effective treatment option that avoids further surgery. In absence of residual tumor on imaging (including suspicious lymph nodes), radiotherapy alone is recommended. In case of residual tumor on imaging, including suspicious lymph nodes, chemoradiotherapy is recommended (grade D).

- Para-aortic lymph node dissection, at least up to inferior mesenteric artery, may be considered in patients without suspicious para-aortic nodes on imaging for staging purposes (grade $\mathrm{C}$ ).

- Debulking of suspicious pelvic lymph nodes may be considered.

- Radical surgery is an option in patients without lymph node involvement on imaging and in the absence of an upfront indication for adjuvant radiotherapy (combination of negative prognostic factors (grade D).

- Pelvic lymph node dissection should be performed as the first step of the surgery. Intraoperative assessment of pelvic lymph nodes may be considered. If intraoperative lymph node assessment is negative or is not performed, radical parametrectomy with the resection of the upper vagina should be performed preferably using minimally invasive techniques. The type of radical parametrectomy (extent of parametrial resection) should be tailored to the presence of prognostic risk factors of the primary tumor as described above (Table 3).

- Complete description of the template used for radical parametrectomy should be present in the operative report.

- The 2017 modification of the Querleu-Morrow classification is recommended as a tool (Table 4).

- If lymph node involvement, including macrometastases or micrometastases, is detected intraoperatively, further surgery (pelvic lymph node dissection and radical parametrectomy) should be avoided, and chemoradiotherapy is recommended (grade D). Para-aortic lymph node dissection, at least up to inferior mesenteric artery, may be considered for staging purposes (grade C). Debulking of suspicious nodes may be considered.

\section{Management of patients with pT1b2 and higher or involved surgical margins or residual tumor including involved lymph node on imaging}

- In patients with stage pT1b2 and higher, involved surgical margins or in those with residual tumor including involved lymph node on imaging, chemoradiotherapy is recommended, and further surgery should be avoided.

- Para-aortic lymph node dissection, at least up to inferior mesenteric artery, may be considered for staging purposes in patients with negative para-aortic lymph nodes on imaging (grade $\mathrm{C}$ ).

- Debulking of suspicious pelvic lymph nodes may be considered.

\section{Management of locally advanced cervical cancer}

\section{Stage $\mathrm{T} 1 \mathrm{~b} 2 / \mathrm{T} 2 \mathrm{a} 2$ and negative lymph nodes on radiological staging}

- Treatment strategy should aim for avoiding the combination of radical surgery and postoperative external radiotherapy because of the significant increase in morbidity and no evident impact on survival (grade B).

- Definitive platinum-based chemoradiotherapy and brachytherapy are the preferred treatment (see PRINCIPLES OF RADIOTHERAPY) (grade A).

- Para-aortic lymph node dissection, at least up to inferior mesenteric artery, may be considered before chemoradiotherapy and brachytherapy. Pelvic lymph node dissection is not required (grade C).

- Radical surgery is an alternative option, in particular in patients without negative risk factors (combinations of tumor size, LVSI, and/or depth of stromal invasion). Quality of surgery, both parametrectomy and lymph node dissection, is, however, of key importance in the management of large tumors. Intraoperative assessment of lymph node status (frozen section) is recommended as the first step. If lymph node involvement is detected intraoperatively, including macrometastases or micrometastases, further pelvic lymph node dissection and radical hysterectomy should be avoided, and patients should be referred for definitive chemoradiotherapy and brachytherapy. Para-aortic lymph node dissection, at least up to inferior mesenteric artery, maybe considered for staging purposes. If intraoperative lymph node assessment is negative or is not done, systematic pelvic lymph node dissection should be performed. Type $\mathrm{C} 2$ radical hysterectomy is recommended. 
- Neoadjuvant chemotherapy followed by radical surgery is a controversial alternative. The benefit of tumor downsizing with regard to prognosis has not been proven (grade C).

\section{Stage T1b2/T2a2 and involved lymph nodes on radiological staging}

- Definitive chemoradiotherapy and brachytherapy are recommended in patients with unequivocally involved pelvic lymph nodes on imaging (see PRINCIPLES OF RADIOTHERAPY) (grade A).

- An additional radiation boost to the involved lymph nodes should be applied (see PRINCIPLES OF RADIOTHERAPY) (grade C).

- Para-aortic lymph node dissection, at least up to inferior mesenteric artery, may be considered before treatment for staging purposes in patients with negative para-aortic lymph node on imaging (grade $\mathrm{C}$ ).

- Debulking of suspicious pelvic lymph nodes may be considered.

\section{Stages $T 2 b, T 3 a / b, T 4 a$}

- Definitive platinum-based chemoradiotherapy and brachytherapy are recommended (see PRINCIPLES OF RADIOTHERAPY) (grade A).

- An additional radiation boost to the involved lymph nodes should be applied (see PRINCIPLES OF RADIOTHERAPY) (grade C).

- Para-aortic lymph node dissection, at least up to inferior mesenteric artery, may be considered before treatment in patients with negative para-aortic lymph nodes on imaging (grade C).

- Debulking of suspicious pelvic lymph nodes may be considered. Pelvic exenteration is an option in selected cases with stage T4 N0 M0 disease.

\section{Cervical stump cancer}

- Management of cervical stump cancer follows the recommendations for patients without previous subtotal hysterectomy. Adaptation of radiotherapy may be necessary, in particular for brachytherapy.

\section{Distant metastatic disease at presentation and recurrent disease}

\section{Distant metastatic disease at presentation}

- Patients with distant metastatic disease at presentation should have a full diagnostic workup (see STAGING) to assess extent of disease, suitability for active treatment, and treatment modality including best supportive care.
- In medically fit patients with widespread distant metastatic disease at presentation (visceral +/- nodal), combination chemotherapy is recommended. Carboplatin/paclitaxel and cisplatin/paclitaxel are preferred regimens in the first-line treatment (grade B).

- Addition of bevacizumab to standard chemotherapy is recommended in patients with good performance status and where the risk of significant gastrointestinal/ genitourinary toxicity has been carefully assessed and discussed with the patient (grade B).

- Patients with limited distant metastatic disease at presentation, confined to the para-aortic lymph node region, should be treated with curative intent with definitive extended field chemoradiotherapy including brachytherapy. Treatment algorithm may also include surgical debulking of enlarged lymph node and additional chemotherapy (grade D).

- Patients with supraclavicular lymph node as the only site of distant disease can be considered for chemoradiotherapy with curative intent. Treatment algorithm may include additional chemotherapy.

- Adjuvant chemotherapy may be considered in cases carrying a high risk of recurrence such as positive margins, positive lymph node, or LVSI-positive tumors (grade C).

- The role of radiotherapy in palliating symptoms such as bleeding and pain must be considered especially in radiotherapy naive patients.

\section{Recurrent disease}

\section{Curative intent treatment}

- Treatment of recurrent disease with curative intent requires centralization and involvement of a broad multidisciplinary team including gynecologic oncologist, radiation oncologist, radiologist, pathologist, medical oncologist, urologist, and plastic surgeon. A structured program for multidisciplinary diagnostic workup, treatment, and follow-up must be present in centers responsible for the treatment.

- Each center involved in the primary treatment of cervical cancer should have an established network for discussion of difficult cases and willingness for referring patients with recurrence for treatment to highly specialized units.

- Participation in clinical trials is encouraged to improve the clinical evidence for the effect of curative treatment of recurrent disease.

\section{Curative intent treatment - diagnostic workup}

- The aim of the diagnostic workup is to exclude distant metastases and locoregional tumor extension beyond curative treatment.

- The recurrence should be confirmed by histological examination. 
- Patients with multiple nodal/distant metastases or multifocal local disease with extensive pelvic wall involvement are usually not considered candidates for curative treatment. The prognostic factors should be carefully evaluated and balanced in relation to the major morbidity caused by the treatment.

- A full diagnostic package consisting of relevant imaging is recommended to establish the status of the disease locally, regionally, and systemically (see STAGING).

- Patient should be carefully counseled regarding not only treatment options but also the involved risks and consequences.

\section{Curative intent treatment - central pelvic recurrence after primary surgery}

- Definitive chemoradiotherapy combined with imageguided adaptive brachytherapy (IGABT) is the treatment of choice (see PRINCIPLES OF RADIOTHERAPY). The use of boost by external beam techniques to replace brachytherapy is not recommended (grade D).

- For brachytherapy, small superficial lesions (ie, $<5-\mathrm{mm}$ thickness) in the vagina may be treated using a vaginal cylinder, ovoids, or mold, whereas other lesions usually require combined intracavitary-interstitial techniques.

\section{Curative intent treatment - pelvic sidewall recurrence after primary surgery}

- Definitive chemoradiotherapy is the preferred option (grade D).

- Extended pelvic surgery may be considered in highly selected patients provided that the tumor does not invade extensively into the pelvic sidewall.

- Combined operative-radiotherapy procedures using intraoperative radiotherapy or brachytherapy are an option if free surgical margins are not achievable (grade D).

- Definitive radiotherapy or chemoradiotherapy followed by a stereotactic ablative boost/image-guided interstitial brachytherapy/particle beam therapy is an emerging option.

\section{Curative intent treatment - central pelvic or pelvic sidewall recurrence after radiotherapy or chemoradiotherapy}

- Pelvic exenteration is recommended for central pelvic recurrence where there is no involvement of the pelvic sidewall and extrapelvic nodes (grade D).

- Laterally extended endopelvic resection may be considered for a recurrence that extends close to or involves the pelvic sidewall.
- Reirradiation with IGABT for central recurrences is an alternative option especially in patients unfit for or refusing exenteration surgery, which should be restricted to highly specialized centers.

\section{Curative intent treatment - role of chemotherapy}

- If further surgery or radiotherapy is considered, no more than 2 to 4 courses of combination chemotherapy should be given to avoid unnecessary long interval before definitive treatment. Locoregional recurrences, which at diagnosis appear incurable, should be reassessed for the possibility of radical treatment if major response is obtained.

- Suitable candidates for adjuvant chemotherapy are patients who recover well within 2 months after primary treatment for recurrence.

\section{Curative intent treatment - nodal and oligometastatic recurrences}

- Localized para-aortic, mediastinal, and/or periclavicular recurrences above previously irradiated fields may be treated by radical external beam radiotherapy (EBRT) if possible in combination with concomitant chemotherapy.

It is recommended to electively irradiate the immediate regional nodal stations below and upstream.

- The therapeutic effect of nodal resection/debulking is unclear and should, if possible, always be followed by radiotherapy.

- The management of isolated organ metastases (lung, liver, etc) should be discussed in a multidisciplinary team involved in the treatment of the specific organ affected by the metastasis and should be treated according to the preferred method for that organ involving local resection, radiofrequency ablation, interventional brachytherapy, or stereotactic ablative radiotherapy according to size and anatomical position.

\section{Palliative treatment}

- Recommendations for palliative treatment should be made only after a thorough review of the case by a specialist multidisciplinary team and taking into account the performance status, comorbidities, patient's symptoms, and wishes of the patient. The palliative care specialist should be actively involved.

- Palliative taxane/platinum combination chemotherapy with/ without bevacizumab is the preferred option (grade B). 
- There is currently no standard second-line chemotherapy, and such patients should be considered for clinical trials.

- In symptomatic patients, palliative treatment should be tailored according to clinical situations.

- In patients with disseminated disease at presentation, radiotherapy (usually a fractionated course) should be considered for effective palliation (grade D).

- Palliative radiotherapy (single fraction/short course) to control bleeding, discharge, and pain due to pelvic disease or bone metastases should be considered (grade D).

- For spinal cord compression due to bone metastases, neurosurgical intervention or short-course fractionated radiotherapy schedule should be considered.

- Surgical interventions including diversion stoma and/or stenting should be considered as appropriate, for example, in case of obstructive symptomatic disease.

\section{Follow-up}

\section{General recommendations}

Primary objectives of follow-up for patients with cervical cancer should include the following:

- Early detection of recurrent disease

- Patient education and support

- Cancer rehabilitation with the goal to prevent and reduce psychosocial, physical, social, and existential consequences of cancer and its treatment starts at time of diagnosis. The efforts should optimize the physical abilities and quality of life of women affected by cervical cancer and include family members/caregivers. Several professions for counseling should be available, for example, psychologist, sexual therapist, physiotherapist, and dietitian.

- Assessment of long-term outcome of novel treatment strategies

- Quality control of care

Each visit should be composed of the following:

- Patient history (including elicitation of relevant symptoms)

- Physical examination (including a speculum examination and bimanual pelvic examination)

- Physician assessment of adverse events using validated scales (eg, Common Terminology Criteria for Adverse Events)

- Prevention and management of cancer-and treatment-related adverse effects, for example, sexual dysfunction (eg, counseling, vaginal lubricants, local estrogen)
- In case of the appearance of treatment-related symptoms, a referral to a dedicated specialist (eg, gastroenterologist, urologist/gynecologist) should be considered.

- Patients should be educated about symptoms of potential recurrence and potential long-term and late effects of treatment. Patients should also be counseled on sexual health, lifestyle adaptation, nutrition, exercise, obesity, and cessation of smoking.

- Follow-up schemes may be individualized, taking into account prognostic factors, treatment modality, and estimated risk and/or occurrence of adverse effects. In general, follow-up intervals of 3 to 4 (6) months for the first 2 years and then 6 to 12 months up to 5 years are recommended (grade C).

- Prescription of hormonal replacement treatment to cervical cancer survivors with premature menopause is advocated and should be according to regular menopausal recommendation. Combined estrogen and progestin replacement therapy should be prescribed if uterus is in situ (including after definitive radiotherapy). Monotherapy with estrogen is recommended after hysterectomy (grade D).

- Imaging and laboratory tests should be performed based on symptoms or findings suggestive of recurrence or morbidity.

- In symptomatic women, MRI or CT should be considered to assess potential clinical recurrence. If positive, wholebody PET-CT should be performed in patients in whom salvage therapy (surgery or radiotherapy) is being considered. Similarly, for suspected recurrence, PET-CT can be added when other imaging finding is equivocal.

- Pathologic confirmation of any persistent or recurrent tumor should be considered. If a lesion is located deeply in the endocervix (in case of conservative treatment or definitive chemoradiotherapy), ultrasound-guided Tru-Cut biopsy is the preferred method. For any disease beyond the primary tumor site, ultrasound or CT-guided methods can be used to achieve pathologic confirmation. In case of clinically or radiologically suspicious disease, a negative biopsy may not be conclusive.

\section{Follow-up after fertility-sparing treatment}

- All women remain at risk of tumor recurrence following FST and must be carefully followed up. Follow-up should be carried out by a provider with specific expertise in detection of lower genital tract dysplasia (eg, gynecologic oncologist, colposcopy expert).

- Follow-up intervals should be 3 to 4 months for the first 2 years postoperatively, and then 6 to 12 months up to 5 years. Thereafter the patient may return to populationbased screening. The duration of follow-up, however, may be individualized depending on the risk of recurrence or persistence of treatment-related complications (grade C). 
- Follow-up should include HPV testing (with or without cytology). Colposcopy in combination with HPV testing in parallel performed by an experienced colposcopist is an option. The incorporation of high-risk HPV testing at 6 , 12 , and 24 months after treatment is advocated. If HPV testing is negative, then every 3 to 5 years as long as follow-up is indicated (grade C).

\section{Follow-up after simple or radical hysterectomy}

- Follow-up should be carried out by physician experienced with follow-up care after surgery following the general recommendations (see above). The vaginal vault cytology is not recommended.

\section{Follow-up after definitive Chemoradiotherapy}

- The same imaging method should be used for evaluation of tumor response as was used at baseline.

- Imaging should be performed not earlier than 3 months following completion of treatment. In dubious cases, a reevaluation should be performed not before 8 weeks thereafter.

- For re-evaluation purposes, the optimal diagnostic workup for local extent is pelvic MRI, and for distant spread, it is chest/abdomen CT or PET-CT (preferred after definitive chemoradiotherapy or in high-risk patients) (grade B).

- Follow-up should be performed by a physician experienced with follow-up care after radiotherapy. Cytology is not recommended in these patients.

- Providers should inform and educate on sexual and vaginal health because vaginal stenosis and dryness may occur. Vaginal dilation should be offered, as well as vaginal lubricants and local estrogen.

\section{Cervical cancer in pregnancy}

- $\quad$ Every patient diagnosed with CCIP must be counseled by a multidisciplinary team. This team should consist of experts in the fields of gynecologic oncology, neonatology, obstetrics, anesthesiology, radiation oncology, medical oncology, psychooncology, and, if requested, theology or ethics.

- Given the large spectrum of described therapeutic options, the multidisciplinary team recommends an individual consensual treatment plan according to patient's intention, tumor stage, and gestational age of pregnancy at cancer diagnosis. Primary aims of recommended treatment plan are oncological safety of the pregnant woman, as well as survival without additional morbidity of the fetus.
- Treatment of patients with CCIP should exclusively be done in gynecologic oncology centers associated with a highest level perinatal center with expertise in all aspects of oncologic therapy in pregnancy and intensive medical care of premature neonates. Because of the low incidence of CCIP, centralization in a few well-equipped facilities is compulsory.

- Besides clinical examination and histologic verification of invasive cervical cancer, preferred imaging modalities for clinical staging in patients with CCIP include MRI or expert ultrasound. Because of limited experience and inherent radioactivity PET-CT (PET-MRI) should be indicated only under very selected circumstances (grade D).

- Tumor involvement of suspicious nodes should be verified histologically because of its prognostic significance and the impact on the management up to 24th week of gestation (fetal viability), preferably by minimally invasive approach.

Depending on tumor stage and gestational week of pregnancy, the following treatment options have to be discussed with the patient including risks and benefits of individual approaches (grade D):

- Adapted surgery including removal of the tumor: conization, trachelectomy, and lymph node staging (see above) according to the stage of the disease with the intent to preserve the pregnancy.

- Radical surgery or definitive chemoradiation as recommended for the stage of the disease without preservation of the pregnancy, with or without previous pregnancy termination.

- Delay of oncological treatment until fetal maturity (if possible 932 weeks of gestation) and beginning of cancerspecific treatment immediately after delivery by cesarean section.

- Chemotherapy until fetal maturity and beginning of cancer-specific treatment immediately after delivery by cesarean section. Treatment after delivery must consider application of previous chemotherapy. In patients with locally advanced stage or with residual tumor after conization that cannot be completely excised (risk of premature rupture of membranes and/or cervical insufficiency), platinum-based chemotherapy can be considered starting earliest at 14 weeks of gestation.

- Spontaneuous delivery seems to have negative prognostic impact in patients with CCIP. Thus, cesarean section after the 32 nd week of gestation (if possible) is the recommended mode of delivery. At the time of or following casarean section, definitive stage-adjusted oncologic therapy has to be performed corresponding to that of nonpregnant women, taking into account therapy that has already been given during pregnancy (grade D). 


\section{Principles of radiotherapy}

\section{Definitive Chemoradiotherapy and brachytherapy: General aspects}

- Definitive management (without tumor related surgery) consists of concomitant pelvic chemoradiotherapy (platinum based) and brachytherapy or pelvic EBRT alone and brachytherapy.

- Overall treatment time for the definitive treatment should not exceed 7 to 8 weeks.

- Delay of treatment and/or treatment interruptions have to be avoided.

\section{Definitive chemoradiotherapy}

- External beam radiotherapy is recommended minimum as 3-dimensional (3D) conformal radiotherapy. The preferred treatment is intensity-modulated radiotherapy (IMRT) because of the more conformal dose distribution that maximizes sparing of organs at risk.

- External beam radiotherapy can be applied as concomitant chemoradiotherapy with total dose of 45 to $50 \mathrm{~Gy}$ (1.8 Gy per fraction) and single-agent radiosensitizing chemotherapy, preferably cisplatin (weekly $40 \mathrm{mg} / \mathrm{m}^{2}$ ) so that definitive radiotherapy is not compromised. If cisplatin is not applicable, alternative treatment options are fluorouracil or carboplatin. External beam radiotherapy may also be applied without concomitant chemotherapy according to treatment selection (ie, patients unfit for any chemotherapy). In such cases, regional hyperthermia may be considered.

- Tumor and lymph node-related target volume for IMRT includes the primary cervical tumor and the adjacent tissues such as parametria, uterine corpus, upper vagina, and the pelvic lymph nodes (obturator, internal, external and common iliac, presacral). In case of pelvic lymph node involvement indicating an increased risk of para-aortic lymph node spread, EBRT may include the para-aortic region up to the renal vessels (45 Gy). In case of paraaortic lymph node involvement, target volume includes at a minimum the region up to the renal vessels.

- A reduced target volume for EBRT resulting in a small pelvic field not including the common iliac nodes may be considered in low-and intermediate-risk T1b1 patients with negative lymph nodes on imaging and no LVSI.

- Boost treatment for involved lymph node(s) may be applied as simultaneous integrated boost within the IMRT treatment or as sequential boost. The total dose including the contribution from brachytherapy should be 55 to 60 Gy (equieffective dose to 2 Gy per fraction [EQD2]). An alternative treatment option is surgical debulking of enlarged nodes.
- Image-guided radiotherapy (IGRT) is recommended for IMRT to ensure safe dose application in the tumor-related targets, to account for motion uncertainties, to reduce margins, and to achieve reduced doses to organs at risk.

- Overall treatment time for EBRT should not exceed 5 to 6 weeks.

\section{Definitive brachytherapy}

- Image-guided adaptive brachytherapy is recommended, preferably using MRI at the time of brachytherapy. Image-guided adaptive brachytherapy is delivered in large tumors toward the end of or after concomitant chemoradiotherapy. Repeated gynecologic examination is mandatory, and alternative imaging modalities such as CT and ultrasound may be used.

- The tumor-related targets for brachytherapy include the residual gross tumor volume (GTV-T $\mathrm{T}_{\text {res }}$ ) after chemoradiotherapy, the adaptive high-risk clinical target volume $\left(\mathrm{CTV}-\mathrm{T}_{\mathrm{HR}}\right)$ including the whole cervix and residual adjacent pathologic tissue, and the intermediate-risk clinical target volume (CTV-T $\mathrm{IR})$.

- Intracavitary and combined intracavitary/interstitial brachytherapy should be performed under anesthesia.

- The brachytherapy applicator should consist of a uterine tandem and a vaginal component (ovoids/ring/mold/combined ring/ovoid). Combined intracavitary/interstitial brachytherapy for adjusting the application further to the individual target should be considered. The vaginal component carries holes for straight or oblique needle guidance into the parametria.

- In case of significant residual disease in the parametrium (as in any extracervical area, eg, vagina, uterine corpus, adjacent organ), this should become part of the CTV-T $\mathrm{TR}_{\mathrm{HR}}$. The brachytherapy application should be a combined intracavitary/interstitial approach in order to achieve a sufficiently high radiation dose in the whole CTV-T $\mathrm{T}_{\mathrm{HR}}$.

- In IGABT, the planning aim should be to deliver a brachytherapy dose of 40 to 45 Gy (EQD2) to reach a total EBRT+ brachytherapy dose of equal to or greater than 85 to 90 Gy EQD2 (D90) (assuming 45 Gy through EBRT) to the CTV-T $\mathrm{HR}_{\mathrm{HR}}$, equal to or greater than $60 \mathrm{~Gy}$ (D98) to the CTV-T ${ }_{\text {IR }}$, and equal to or greater than $90 \mathrm{~Gy}$ (D98) to the GTV-T $\mathrm{T}_{\text {res. }}$. Three-dimensional and 2D dose volume and point constraints for rectum, bladder, vagina, sigmoid, and bowel are recommended, and they have to be based on the published clinical evidence.

- Point A dose normalization should be used as starting point for stepwise treatment plan optimization, although point A dose reporting and prescription have been extended by the volumetric approach. 
- Brachytherapy should be delivered in several fractions as high dose rate (usually $3-4$ ) or in 1 to 2 fractions as pulse dose rate brachytherapy.

- In large tumors, brachytherapy should be delivered within 1 to 2 weeks toward the end of or after chemoradiotherapy. In limited-size tumors, brachytherapy may start earlier during chemoradiotherapy.

- For the tumor-related targets $\left(\mathrm{GTV}-\mathrm{T}_{\mathrm{res}}, \mathrm{CTV}-\mathrm{T}_{\mathrm{HR}}\right.$, CTV-T $\mathrm{T}_{\mathrm{IR}}$ ), the use of external beam therapy for giving extra dose (eg, parametrial boost, cervix boost) is discouraged, even when using advanced EBRT technology such as stereotactic radiotherapy. The use of a midline block for boosting the parametrium is discouraged when applying advanced IGRT, in particular beyond 45 to $50 \mathrm{~Gy}$.

- Care should be taken to optimize patient comfort during (fractionated) brachytherapy. Preferably this includes a multidisciplinary approach.

\section{Adjuvant radiotherapy or chemoradiotherapy}

- Adjuvant radiotherapy or chemoradiotherapy follows analog principles for target selection and dose and fractionation as outlined for definitive treatment.

- The application of IMRT and IGRT is to be considered as treatment-related morbidity may be reduced.

- Adjuvant (additional) brachytherapy should be considered only if a well-defined limited area - accessible through a brachytherapy technique - is at high risk of local recurrence (eg, vagina, parametrium). Such adjuvant brachytherapy should follow the major principles outlined above for image-guided brachytherapy.

\section{Definitive 3D conformal EBRT or chemoradiotherapy- and radiography-based brachytherapy}

- Three-dimensional conformal radiotherapy alone or as definitive concomitant chemoradiotherapy (platinum based) \pm para-aortic radiotherapy and/or 2D radiography-based brachytherapy is recommended, if IMRT and/or IGABT are not available.

- In case of 3D conformal radiotherapy and/or radiographybased brachytherapy, the recommendations for EBRT and IGABT as outlined above in regard to target, dose, fractionation, and overall treatment time have to be respected as much as possible.

- A sequential lymph node boost is applied as appropriate after completion of 3D EBRT.

- Planning aim for brachytherapy should be based on point A. Dose to point A should be equal to or greater than 75 Gy (EQD2) in limited width adaptive CTV-T $\mathrm{HR}_{\mathrm{HR}}(\leq 3$ $\mathrm{cm}$ ) and should aim at higher doses in large width adaptive
$\mathrm{CTV} \mathrm{T}_{\mathrm{HR}}(>4 \mathrm{~cm})$. In addition, dose for the maximum width of the adaptive CTV-T $\mathrm{T}_{\mathrm{HR}}$ should be reported. Radiography-based dose point constraints - plus 3D dose volume constraints as available - for rectum, bladder, vagina, sigmoid, and bowel are recommended, and they have to be based on the published clinical evidence.

\section{Principles of pathological evaluation}

\section{Requirements for specimen submitted for pathological evaluation}

1. Patient information, previous cervical cytology, histological specimens, clinical and radiological data, and colposcopic findings need to be included on the specimen request form.

2. Details of cytology, biopsy, and surgical specimen (cone/ loop specimen, trachelectomy, type of hysterectomy, presence of ovaries and fallopian tubes, presence of lymph nodes and designation of the lymph node sites, presence of vaginal cuff, and presence of parametria) need to be itemized in the specimen request form.

3. Biopsies and surgical specimens should be sent to the pathology department in a container with liquid fixative ("clamping" of the specimen on cork may be done).

4. Cytology specimens should be sent to the pathology department either as a smear preparation (exfoliative cytology on a clearly designated and identifiable slide with patient's name and birth date) or as liquid-based cytology. The latter is necessary when an HPV test is requested.

5. Cone/loop specimen should ideally be sent intact with a suture to identify the 12-o'clock position.

\section{Specimen grossing and sampling}

- Small biopsy specimens should be enumerated and measured.

- The diameter (2 dimensions) and depth of cone/loop specimens should be measured. It should be recorded if the specimen is complete or fragmented. If more than 1 piece of tissue is received, every piece should be measured in 3 dimensions and entirely examined.

- Inking of the surgical margins of cone/loop specimens is optional.

- Dissection of cone/loop specimens should be performed in an appropriate fashion. All the pieces submitted should be in consecutive numerical order. This is important because if tumor is present in more than 1 piece, it needs to be known whether these are consecutive pieces and thus a single tumor or represents multifocal tumor. It is recommended to place only 1 piece of tissue in each cassette. 
There are also techniques that allow embedding of more than 1 piece in a cassette if they are small enough. In cases that do not comprise intact cone/loops, serial radial sectioning and placing of each slice of tissue in a single cassette should be performed.

- The description of the specimen (hysterectomy, trachelectomy, presence of ovaries and fallopian tubes, presence of lymph nodes and indication of the lymph node sites, presence of vaginal cuff, presence of parametria) should be recorded and checked for consistency with the description given in the specimen request form.

- The presence of any gross abnormality in any organ should be documented.

- The dimensions of the uterus for a hysterectomy specimen and the cervix for a trachelectomy specimen should be documented.

- The minimum and maximal length of the vaginal cuff should be documented.

- The size of the parametria should be documented in 2 dimensions (vertical and horizontal).

- Gross tumor involvement of the parametrium, vagina, uterine corpus, or other organs should be documented. The relationship of the cervical tumor to the vaginal and parametrial margins (and upper margin in case of a trachelectomy specimen) should be measured and appropriate sections taken to demonstrate this.

- Parametrial and vaginal margins should be inked.

- Parametria should be submitted totally for histological examination.

- The upper surgical margin of a trachelectomy specimen should be inked.

- The upper margin of a trachelectomy specimen should be sampled in its entirety in a way that demonstrates the distance of the tumor to the margin. The vaginal margin should be examined totally as radial sections if no tumor is seen grossly.

- When the tumor is small (or with tumors that cannot be identified macroscopically), the cervix should be separated from the corpus, opened and processed as for a cone/loop specimen.

- In the case of a large tumor, the hysterectomy or trachelectomy specimen should be opened in the sagittal plane.

- The description of the cervix and measurement of any gross tumor mass should be documented.

- Gross tumors should be measured in 3 dimensions, namely, 2 measurements of horizontal extent and the depth of invasion.

- The tumor site within the cervix should be documented.

- The cervical tumor should be sampled in order to demonstrate the maximum depth of invasion, the relationship of the tumor with the surgical borders, and the extension to other organs.

- If visible, the site of a previous cone biopsy should be documented.
- At least 1 block per centimeter of the greatest tumor dimension for large tumors should be taken.

- Additional blocks including the cervix adjacent to the tumor should be taken in order to demonstrate precursor lesions.

- The whole cervix should be sampled in the case of a small tumor or where no macroscopic tumor is identified.

- The uterine corpus, vagina, and adnexa should be sampled according to standard protocols if not involved by tumor. If the uterine corpus and/or adnexa are grossly involved, additional blocks should be sampled.

- The entire vaginal margin should be blocked.

- All the lymph nodes should be submitted for histological examination. If the lymph nodes are grossly involved, representative samples are sufficient. If grossly uninvolved, each node should be sliced at 2-mm intervals and totally embedded. From each block, hematoxylineosin (H\&E) sections should be taken. Lymph nodes should be submitted in separate cassettes according to the site recorded on the specimen request form.

\section{Pathological analysis of sentinel lymph node}

- Intraoperative assessment should be performed on a grossly suspicious sentinel node and may be performed on a "nonsuspicious" sentinel lymph node(s) because the confirmation of tumor involvement will result in abandoning a hysterectomy or trachelectomy.

- For intraoperative evaluation, the sentinel lymph node(s) needs to be sent to the pathology department in a container without liquid fixative.

- Intraoperative analysis requires gross dissection of the resected adipose tissue by the pathologist with the selection of the lymph node(s).

- For a lymph node with obvious gross tumor, a single section is adequate for frozen section.

- Frozen section may be combined with imprint cytology.

- Any nonsuspicious sentinel node should be bisected (if small) or sliced at 2-mm thickness and entirely frozen.

- From each sample, histological sections should be cut and stained by $H \& E$.

- After frozen section analysis, the tissue should be put into a cassette, fixed in liquid fixative and subsequently processed and embedded in paraffin.

- Sentinel lymph node(s) tissue blocks should be entirely analyzed by examining multiple serial sections at different levels with H\&E stains. Cytokeratin stains should be performed on all blocks.

- The detection of micrometastases and isolated tumor cells should be improved by immunohistochemistry with pancytokeratinantibodies (eg, AE1/AE3). Different procedures have been published, and there is no standard method. Cytokeratin-positive cells should always be 
correlated with the morphology. Müllerian inclusions (endosalpingiosis, endometriosis) and mesothelial cells may rarely be present in pelvic and para-aortic lymph nodes and be cytokeratin positive.

\section{Requirements for pathology report}

- Description of the specimen(s) submitted for histological evaluation.

- Macroscopic description of specimen(s) (biopsy, loop/cone, trachelectomy, hysterectomy) including specimen dimensions ( 3 dimensions), number of tissue pieces for loop/cones, and maximum and minimum length of vaginal cuff and the parametria in 2 dimensions.

- Macroscopic tumor site(s), if the tumor is visible grossly, in trachelectomy and hysterectomy specimens.

- Tumor dimensions including 2 measurements of horizontal extent and depth of invasion or thickness (tumor dimension should be based on a correlation of the gross and histological features). When multifocal separate tumors are present, each should be described and measured separately, and the largest used for tumor staging. Specimens from prior conization and subsequent conization, trachelectomy, or hysterectomy should be correlated for estimation of the tumor size. This is of importance because different specimens may have been reported at different institutions. It should also be recognized that simply adding the maximum tumor size in separate specimens may significantly overestimate the maximum tumor dimension.

- Histological tumor type and tumor grade.

- The presence or absence of LVSI.

- Coexisting pathology (squamous intraepithelial lesion/ cervical intraepithelial neoplasia, adenocarcinoma in situ, stratified mucin-producing intraepithelial lesion).

- Minimum distance of uninvolved cervical stroma.

- Margin status (invasive and preinvasive diseases). Specify the margin(s).

- Lymph node status including sentinel lymph node status, the total number of nodes found and the number of positive lymph nodes, and the presence of extranodal extension (list for all separates sites). Micrometastasis (>0.2 $\mathrm{mm}$ and up to $2 \mathrm{~mm}$ ) are reported as $\mathrm{pN} 1(\mathrm{mi})$. Isolated tumor cells no greater than $0.2 \mathrm{~mm}$ in regional nodes should be reported as pN0 (i+).

- Pathologically confirmed distant metastases.

- Provisional pathological staging pretumor board/ multidisciplinary team meeting (American Joint Committee on Cancer, eighth edition).

Items to be included on pathology report of carcinomas of the cervix

\begin{tabular}{|c|c|c|}
\hline Clinical/Surgical & Macroscopic & Microscopic \\
\hline Specimen(s) submitted & $\begin{array}{l}\text { Specimen dimensions } \\
\text { - No. tissue pieces (for loops/cones) } \\
\text { - Tissue piece dimensions (for loops/cones) } \\
\text { Diameter of ectocervix (2 measurements) } \\
\text { Depth of specimen } \\
\text { - Vaginal cuff } \\
\text { Minimum length } \\
\text { Maximum length } \\
\text { - Size of parametria in } 2 \text { dimensions } \\
\text { (vertical and horizontal) } \\
\text { Macroscopic tumor site(s) }\end{array}$ & $\begin{array}{l}\text { Tumor dimensions* } \\
\text { - Horizontal extent ( } 2 \text { measurements) } \\
\text { - Depth of invasion or thickness } \\
\text { Histological tumor type } \\
\text { Histological tumor grade } \\
\text { LVSI } \\
\text { Coexisting pathology } \\
\text { - Squamous intraepithelial lesion/cervical intraepithelial neoplasia } \\
\text { - Adenocarcinoma in situ } \\
\text { - Stratified mucin-producing intraepithelial lesion } \\
\text { Minimum distance of uninvolved cervical stroma } \\
\text { Margin status (invasive and preinvasive diseases). } \\
\text { Specify the margin(s) } \\
\text { Lymph node status (sentinel lymph node status, } \\
\text { number involved/number retrieved and presence } \\
\text { of extranodal extension) } \\
\text { Pathologically confirmed distant metastases } \\
\text { Pathological staging pretumor board/multidisciplinary } \\
\text { team meeting (TNM category) }\end{array}$ \\
\hline
\end{tabular}

*Tumor dimension should be based on a correlation of the gross and histological features. 
Acknowledgments The authors thank the Guidelines, Recommendations and Quality Assurance Committee of ESGO. The authors also thank the 159 international reviewers for their participation (Supplementary Material 3). Lastly, the authors also wish to express gratitude to the Institut National du Cancer (France) for providing major funding for this work.

\section{Compliance with ethical standards}

Conflict of interest The authors declare no conflicts of interest.

\section{Affiliations}

David Cibula $^{1} \cdot$ Richard Pötter $^{2} \cdot$ François Planchamp ${ }^{3}$ - Elisabeth Avall-Lundqvist ${ }^{4}$ • Daniela Fischerova ${ }^{1}$. Christine Haie-Meder ${ }^{5}$. Christhardt Köhler ${ }^{6}$. Fabio Landoni ${ }^{7}$. Sigurd Lax ${ }^{8}$. Jacob Christian Lindegaard ${ }^{9}$. Umesh Mahantshetty ${ }^{10} \cdot$ Patrice Mathevet $^{11} \cdot$ W. Glenn McCluggage ${ }^{12} \cdot$ Mary McCormack $^{13} \cdot$ Raj Naik $^{14}$. Remi Nout ${ }^{15}$. Sandro Pignata ${ }^{16}$. Jordi Ponce ${ }^{17}$. Denis Querleu ${ }^{3}$. Francesco Raspagliesi ${ }^{18}$. Alexandros Rodolakis ${ }^{19} \cdot$ Karl Tamussino $^{20}$ - Pauline Wimberger ${ }^{21}$ - Maria Rosaria Raspollini ${ }^{22}$

1 Gynecologic Oncology Center, Department of Obstetrics and Gynecology, First Faculty of Medicine, Charles University and General University Hospital, Kateřinská 32, 121 08 Prague, Czech Republic

2 Department of Radiotherapy, Medical University of Vienna, Vienna, Austria

3 Institut Bergonié, Bordeaux, France

4 Linkoping University, Linkoping, Sweden

5 Department of Radiotherapy, Institut Gustave Roussy, Villejuif, France

6 Medical Faculty, Department of Gynecology, Asklepios Hambourg Altona and University of Cologne, Hamburg, Germany

7 University of Milan Bicocca, Monza, Italy

8 General Hospital Graz Sued-West, Graz, Austria

9 Department of Oncology, Aarhus University, Aarhus, Denmark

10 Department of Radiation Oncology, Tata Memorial Hospital, Mumbai, India
11 Lausanne University, Lausanne, Switzerland

12 Department of Pathology, Belfast Health and Social Care Trust, Belfast, UK

13 University College Hospital London, London, UK

14 Queen Elizabeth Hospital, Gateshead, UK

15 Department of Radiation Oncology, Leiden University, Leiden, The Netherlands

16 Istituto Nazionale per lo Studio e la Cura dei Tumori "Fondazione G. Pascale," IRCCS, Naples, Italy

17 University Hospital of Bellvitge (IDIBELL), Barcelona, Spain

18 Fondazione IRCCS Istituto Nazionale Tumori, Milan, Italy

19 Athens University, Athens, Greece

20 Medical University of Graz, Graz, Austria

21 Dresden University, TU Dresden, Dresden, Germany

22 University Hospital, Careggi, Florence, Italy 